American Journal of Applied Sciences 4 (11): 925-931, 2007

ISSN 1546-9239

(C) 2007 Science Publications

\title{
Orientation Detection of Sensed Objects using a Four Sensor Tactile System with Applications in Bio-Robotics
}

\author{
${ }^{1}$ Ashkan Mirbagheri, ${ }^{1}$ Javad Dargahi, ${ }^{2}$ Siamak Najarian and ${ }^{3}$ Farhad Tabatabai Ghomshe \\ ${ }^{1}$ Concordia University, Department of Mechanical and Industrial Engineering, 1455 de Maisonneuve \\ Blvd. West, Montreal, Quebec, Canada \\ ${ }^{2}$ Department of Biomechanics, Artificial Tactile Sensing and Robotic Surgery Lab, \\ Faculty of Biomedical Engineering, Amirkabir University of Technology, Tehran, Iran \\ ${ }^{3}$ University of Social Welfare \& Rehabilitation Sciences, Tehran, Iran
}

\begin{abstract}
A novel approach is presented that can be used in determining the sensitivity of a four sensor tactile sensor to the orientation of the contacted objects. Computer simulations were conducted by using a rectangular probe of $14 \mathrm{~mm}$ by $7 \mathrm{~mm}$ size and applying three magnitudes of forces, i.e., 0.5 , 1 , and $2 \mathrm{~N}$. A bi-variate function was obtained, showing the amount of output charge against both the angle of orientation and the magnitude of the applied force. By computing the errors, the proposed model proved efficient in predicting the orientation of sensed objects with reasonable accuracy.
\end{abstract}

Keywords: Sensing Elements, Tactile Sensing, Bio-Robotics, Mathematical Modeling, PVDF

\section{INTRODUCTION}

Endoscopic or laparoscopic surgeries are among the most popular kinds of minimally invasive surgeries (MIS) which have been developed to decrease the traumatic effects of various types of surgeries ${ }^{[1-5]}$. In this kind of operative procedure and unlike open surgeries, the surgeons cannot use their hands directly in manipulating the operation site. In other words, they do not have their hands inside the patient's body ${ }^{[6-9]}$. This means that various medical manipulations are not being performed in the operation sites. Therefore, the surgeons would have to use long slender instruments outside the operative zone and transmit the manipulations, such as grasping, cutting or other maneuvers to the sites by the hand tools ${ }^{[10-12]}$. Although MIS has a considerable number of advantages, such as, less tissue damage, less postoperative pain, faster recovery period, fewer postoperative complications, and reduced hospital stay, it also suffers from several disadvantages ${ }^{[13-15]}$. These disadvantages include: loss of tactile sensing feedback, the need for increased technical expertise, a possibly longer duration of the surgery, and difficult removal of bulky organs. The loss of tactile sensing feedback translates to the fact that the surgeons would not be able to feel the tissues. For instance, if there are any lumps or abnormal tissues in the background tissue, there is a possibility that the operators might easily miss them. At the same time, the risk of accidental cutting of the surrounding veins or arteries that are embedded in the tissues can lead to serious complications. Due to these problems, the loss of tactile sensing has proven to be a serious issue and has been the target of extensive research ${ }^{[16-20]}$. Some of the main previous research activities on this topic are presented below.

The concept of an inexpensive PVDF pyroelectric radiation sensor of large aperture is described and the design details of the pyroelectric sensor based on the PVDF polymer are given ${ }^{[21]}$. A micro-mechanical sensing platform is proposed with which the various elastic properties of the ovum can be characterized using uniaxial measurements ${ }^{[22]}$. Novel textiles are developed using conducting polymer coatings which are deposited on a foam substrate ${ }^{[23]}$. The resulting structure is sensitive to pressure exerted from all three dimensions making it attractive for use as wearable sensors for medical applications.

The main incentive for conducting the current research was due to the various problems associated with commercial types of tactile sensors. Other researchers have reported issues such as cross-talk between sensing elements, fragility, and complexity, but so far not many practical methods have been

Corresponding Author: $\quad$ Full-Professor of Biomedical Engineering, Department of Biomechanics, Artificial Tactile Sensing and Robotic Surgery Lab, Faculty of Biomedical Engineering, Amirkabir University of Technology, Tehran, Iran, P.O. Box 15875-4413. Tel: (+98-21)-6454-2378. Fax: (+98-21)-66468186 
suggested to handle these liabilities ${ }^{[8]}$. To approach this and following our previous work on the design and manufacturing of a tactile sensor with only four sensing elements, we are now proposing a novel system for detecting the orientation of a sensed object ${ }^{[7]}$. In short, FEM is being employed to predict the details of the tactile system.

\section{MATERIALS AND METHODS}

Modeling of orientation detection: ANSYS software was used for modeling of the sensed object orientation recognition. This analysis enables the user to input load or deflection and directly obtain the generated output charges developed on the surfaces of electrodes. Clearly, the main point of interest in studying this sensor is investigating its sensitivity to orientation of an object. If the object in contact with the sensor area has sharp corners or irregularities, e.g., a rectangle or a triangle, then the outputs of the sensor depend not only on the location of the object but also on its orientation with respect to the sensing elements. A number of simulations were implemented with a rectangular 14 $\mathrm{mm}$ by $7 \mathrm{~mm}$ probe as follows: 1) Under a predetermined load, the rectangular probe is rotated. Then, the responses, or output charges versus rotation angles, are plotted. 2) Normalization of the plots and inverse relations are investigated and attempts are made toward finding some information about the force such as its magnitude, the angle of rotation of the probe. 3) At a specific angle, for instance $0^{\circ}$, loads with different magnitudes are applied. Then, the plots of output charges versus loads are produced.

Rotating the probe under fixed magnitudes of force: The force is distributed over the area of the rectangular probe to create a uniform pressure. Then, the probe is rotated between $0^{\circ}$ to $180^{\circ}$ with respect to the $x$-axis, namely, the drawn direction, in increments of $10^{\circ}$. This is illustrated in Fig. 1.

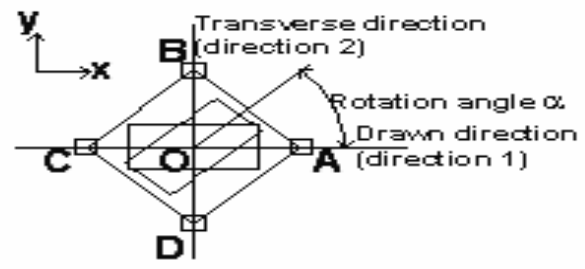

Fig.1: Schematic representation of rotating a rectangular probe.

The letter $O$ in the Fig. represents the center of the circle, as well as the centroid of the rectangular probe.
This simulation is repeated three times for forces with the different magnitudes of $0.5,1$, and $2 \mathrm{~N}$. The SHELL 63 element was selected for meshing the membrane area in FEM.

\section{RESULTS AND DISCUSSION}

In Figs. 2 to 4 , the output charge versus angle of rotation for electrodes $A$ and $B$ is presented when force is $0.5,1$, and $2 \mathrm{~N}$, respectively.

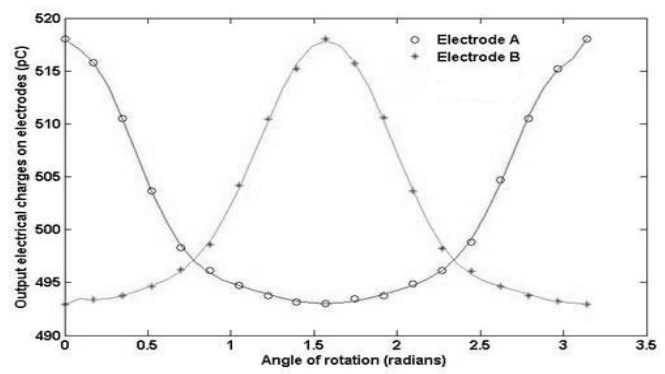

Fig. 2: Charge versus angle of rotation for electrode $A$ and $B$ when force is $0.5 \mathrm{~N}$.

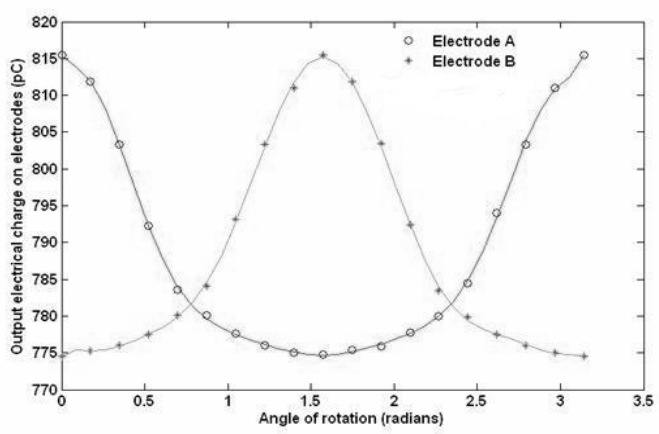

Fig. 3: Charge versus angle of rotation for electrodes $A$ and $B$ when force is $1 \mathrm{~N}$.

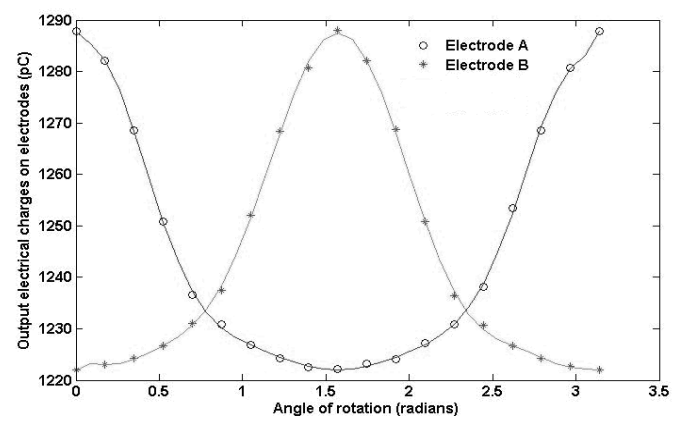

Fig. 4: Charge versus angle of rotation for electrodes $A$ and $B$ when force is $2 \mathrm{~N}$.

It is evident that the trend in the variation of charge against angle of rotation is a combination of descend and ascend for both electrodes. As it is clear in all the above three Figs., for electrode $A$, there is a unique 
behavior: the charge has its maximum amount at an angle of $0^{\circ}$ and as the probe keeps rotating toward an angle of $90^{\circ}$, its charge follows a declining trend and reaches its minimum amount at $90^{\circ}$ angle. Then, it starts increasing until it peaks again at $180^{\circ}$. As a result, the probe is cable of distinguishing the object orientation. The nature of this behavior remains the same for all magnitudes of loads. Understandably, however, there are differences in the range of variation of the output charge and its minimum and maximum values. Likewise, for electrode $B$, there is a unique type of behavior for all loads. However, this time it is exactly opposite to that of electrode $A$. For electrode $B$, the output charge starts from a minimum at $0^{\circ}$ and peaks at $90^{\circ}$, then decreases to its original amount at $180^{\circ}$. One thing that was expected was that, as the sharp corner of the rectangular probe gets closer or points more toward each electrode, the amount of accumulated charge on that electrode increases. This can be attributed to the stress concentration effect; the closer the sharp corner gets to the electrode edge, the higher the stress concentration becomes and therefore the produced charge increases. This is clearly seen in all the Figs and is in accordance with the results obtained for a 4-element sensor ${ }^{[7]}$. An interesting point here is that, each pair of curves intersects with each other at two points. These two points correspond to the rotational angles of $45^{\circ}$ and $135^{\circ}$, respectively. Considering the top view of the entire model and the way the angle of rotation is defined, this is expected.

Results of rotation under a force of $1 \mathrm{~N}$ : Table 1 shows how the charges on all of the four electrodes vary under a constant force of $1 \mathrm{~N}$ with rotation of the probe.

Table 1: Variation of charges versus angle of rotation for all 4 electrodes when force is 1 $\mathrm{N}$ (only some of the data are shown)

\begin{tabular}{ccccc}
\hline Electrode & $0^{\circ}$ & $40^{\circ}$ & $80^{\circ}$ & $170^{\circ}$ \\
\hline$A$ & 815.4188 & 783.5395 & 774.9705 & 810.9745 \\
$B$ & 774.5725 & 780.0890 & 810.9675 & 775.0339 \\
$C$ & 815.4861 & 783.4222 & 774.9639 & 810.9475 \\
$D$ & 774.6369 & 780.0778 & 810.9844 & 775.0733 \\
\hline
\end{tabular}

Now for verifying the existence of an inverse relation the $1 \mathrm{~N}$ force is taken as an example. In this case, the following equation is derived between output charge " $c$ " and the angle of rotation " $a$ " for electrode $A$ : $c_{A}=4.6045 a^{10}-71.577 a^{9}+472.81 a^{8}-1728.4 a^{7}+3808.1 a^{6}-5141.5 a^{5}+$

$4092.9 a^{4}-1688.3 a^{3}+242.11 a^{2}-28.251 a+815.41$

This is of course a non-linear relation between the charge and the angle of rotation, governed by a 10th degree polynomial. The angle (i.e., $a$ ) is in radians. As the first try, the charge was assumed to be $810 \mathrm{pC}$. The obtained angle will be $0.226171 \mathrm{rad}$. Upon running a simulation with this angle (which is $12.959^{\circ}$ ) and a force of $1 \mathrm{~N}$, the charge was $810.397 \mathrm{pC}$ which, compared to the $810 \mathrm{pC}$, corresponds to an error of
$0.049 \%$. This is an acceptable error. By referring to Table 1 and looking at the previous figures, it is noticed that there is not such a big difference in recorded data from electrode $A$ to $C$. The same is true for electrodes $B$ and $D$. As a result, electrodes $B$ and $D$ could be ignored. So, just studying electrodes $A$ and $B$, at least for the case of $1 \mathrm{~N}$ force, suffices. For electrode $B$, the relation between charge and rotational angle is derived as:

$c_{B}=-4.3112 a^{10}+68.534 a^{9}-456.43 a^{8}+1648.7 a^{7}-3498.2 a^{6}$

$+4431.8 a^{5}-3299.4 a^{4}+1393.3 a^{3}-295.58 a^{2}+27.241 a+774.57$

in which $c_{B}$ means charge on electrode $B$. The main obstacle here is having an educated guess on what charge on electrode $B$ appears when charge on element $A$ is $800 \mathrm{pC}$. In all of the simulations, the data for all four elements are recorded; so it is easy to have both of the charges at the same time. However, here it is virtually impossible to make smart selection of pairs of charges for electrodes $A$ and $B$. No clear clue exists as to what the charge on electrode $B$ will be when the charge on electrode $A$ is a certain amount and vice versa. That, in turn, enforces the selection of just one element at time; hence, electrode $A$ is chosen. As the second try, it is assumed that the measured charge on electrode $A$ is $800 \mathrm{pC}$. Then, the charge will be $800 \mathrm{pC}$ and the angle of rotation is $0.3997 \mathrm{rad}$ or $22.901^{\circ}$. A new simulation was performed in ANSYS, under exactly the same conditions, but with this new angle of rotation. Charge on electrode $A$ was calculated to be $801.553 \mathrm{pC}$, with an error of $0.194 \%$. So, this was also acceptable. As the third try, this time the charge was assumed to be $785 \mathrm{pC}$. The obtained angle of rotation is $0.671085 \mathrm{rad}$ or $38.445^{\circ}$. Running a new simulation yields an output charge of $783.666 \mathrm{pC}$ which corresponds to an error of $0.17 \%$, which is relatively small and negligible. As the fourth try, this time the charge was assumed to be 775 pC. Substituting this value in the equation yields an angle of $1.319616 \mathrm{rad}$ or $75.608^{\circ}$. The associated charge obtained from the simulation was $775.398 \mathrm{pC}$, which amounts to an error of $0.051 \%$. In this case, the error has even decreased, and is quite negligible. A summary of the preceding results is presented below in Table 2 .

Table 2: Summary of the estimation errors for a force of $1 \mathrm{~N}$

$\begin{array}{cccc}\begin{array}{c}\text { Assumed charge on } \\ \text { electrode } A(\mathrm{pC})\end{array} & 810 & 800 & 785 \\ \begin{array}{c}\text { Calculated angle of } \\ \text { rotation }\left(^{\circ}\right)\end{array} & 11.176 & 22.901 & 38.445 \\ \begin{array}{c}\text { Calculated charge on } \\ \text { electrode } A(\mathrm{pC})\end{array} & 810.363 & 801.553 & 783.666 \\ \begin{array}{c}\text { Associated error in } \\ \text { guessing the angle }(\%)\end{array} & 0.049 & 0.194 & 0.17\end{array}$

As noticed above, in all cases, the estimation error is very low and thus ignorable. Therefore, it can be 
concluded that interpolation using polynomials serves very well, at least for computing the rotational angle.

Results of rotation under a force of $0.5 \mathrm{~N}$ : Next series of simulations were performed with an applied force of $0.5 \mathrm{~N}$ and rotating the probe in the same manner from $0^{\circ}$ to $180^{\circ}$ at the same location. The equation governing the variation for this case is:

$c_{A}=2.8857 a^{10}-44.854 a^{9}+296.24 a^{8}-10826 a^{7}+2384.5 a^{6}$

$-3217.5 a^{5}+2559.2 a^{4}-1054.7 a^{3}+151.52 a^{2}-17.685 a+517.98$

As the first try, the above equation was set equal to 510 pC. Then angle " $a$ " will be $0.359831 \mathrm{rad}$ or $20.617^{\circ}$. A simulation was conducted with a $0.5 \mathrm{~N}$ load and this rotational angle. The resulting charge on element $A$ was $509.333 \mathrm{pC}$. This results in an acceptable error of $0.131 \%$. Following the same procedure as before, the summary of the estimation errors for a force of $0.5 \mathrm{~N}$ is shown in Table 3.

Table 3: Summary of the estimation errors for a force of $0.5 \mathrm{~N}$

\begin{tabular}{cccc}
\hline $\begin{array}{c}\text { Assumed charge } \\
\text { on electrode } A \\
\text { (pC) }\end{array}$ & 510 & 502.5 & 495 \\
$\begin{array}{c}\text { Calculated angle of } \\
\text { rotation }\left({ }^{\circ}\right)\end{array}$ & 20.617 & 31.796 & 57.158 \\
$\begin{array}{c}\text { Calculated charge } \\
\text { on electrode } A \\
\text { (pC) }\end{array}$ & 509.333 & 501.700 & 494.874 \\
$\begin{array}{c}\text { Associated error in } \\
\text { guessing the angle } \\
(\%)\end{array}$ & 0.131 & 0.159 & 0.025 \\
\hline
\end{tabular}

Once again as tabulated above, in all cases, the estimation error is very low. Now, with the above data in mind, it is suggested to normalize the alreadydeveloped equations and introduce lookup tables and curves. This is described in the next section.

Normalization of the charge versus rotational angle: Normalization was begun with Equation 1, which demonstrates the variation of charge versus angle of rotation when the load is $1 \mathrm{~N}$. Dividing all the coefficients in this equation by 815.41 leads into:

$c_{A}=(5.647 e-3) a^{10}-0.088 a^{9}+0.580 a^{8}-2.120 a^{7}+4.670 a^{6}-6.305 a^{5}$

$+5.019 a^{4}-2070 a^{3}+0.297 a^{2}-0.035 a+1$

When the load is $0.5 \mathrm{~N}$, we have:

$c_{A}=28857 a^{10}-44.854 a^{9}+296.24 a^{8}-10826 a^{7}+2384.5 a^{6}$

$-3217.5 a^{5}+2559.2 a^{4}-1054.7 a^{3}+151.52 a^{2}-17.685 a+517.98$

The normalized relation will be:

$c_{A}=(5.57 k-3) a^{10}-0.087 a^{9}+0.572 a^{8}-2090 a^{7}+4.603 a^{6}-6.212 a^{5}$

$+4.941 a^{4}-2036 a^{3}+0.293 a^{2}-0.034 a+1$
Finally, for the $2 \mathrm{~N}$ load case, the normalized equation is:

$$
\begin{aligned}
& c_{A}=(5.684 e-3) a^{10}-0.088 a^{9}+0.584 a^{8}-2.134 a^{7}+4.702 a^{6}-6.349 a^{5} \\
& +5.054 a^{4}-2083 a^{3}+0.297 a^{2}-0.034 a+1
\end{aligned}
$$

The target of normalization was to seek an equation in the following format:

$Y=A_{1} a^{10}+A_{2} a^{9}+A_{3} a^{8}+A_{4} a^{7}+A_{5} a^{6}+A_{6} a^{5}+A_{7} a^{4}$

$+A_{8} a^{3}+A_{9} a^{2}+A_{10} a+A_{11}$

in which $A_{11}=1$, and $Y$ is the normalized output charge developed on electrode $A$, for a range of forces and rotational angles varying from $0^{\circ}$ to $90^{\circ}$. Then, the above equation would have coupled it to a lookup table such as Table 4.

Table 4: An example of a suggested look-up table

\begin{tabular}{ccc}
$\begin{array}{c}\text { Range of } \\
\text { output charge } \\
\text { of electrode } \\
\text { A (pC) }\end{array}$ & $\begin{array}{c}\text { Factor for } \\
\text { scaling down } \\
\text { the coefficients } \\
\text { of polynomial }\end{array}$ & $\begin{array}{c}\text { Corresponding } \\
\text { normal force (N) }\end{array}$ \\
\hline $\begin{array}{c}493.051 \text { to } \\
517.993\end{array}$ & 517.98 & 0.5 \\
774.7457 to & 815.41 & 1 \\
815.4188 & 1287.8 & 2 \\
1220 to 1290 & & \\
\hline
\end{tabular}

By taking the average of $A_{i}$ coefficient for the previous three equations, the following normalized equation is derived:

$c_{A}=(5.62 e-3) a^{10}-0.088 a^{9}+0579 a^{8}-2115 a^{7}+4.658 a^{6}-6.289 a^{5}$

$+5.005 a^{4}-2063 a^{3}+0.296 a^{2}-0.034 a+1$

The above equation should be used in conjunction with Table 4. In Fig. 5, the variation of the produced charge developed on electrode $A$ versus the applied force is shown when the load on the probe changes in magnitude (provided that the probe is not rotated or the angle of rotation is $0^{\circ}$ ).

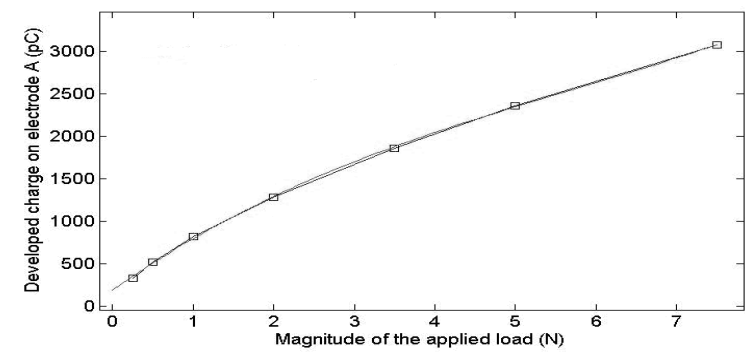

Fig. 5: Curve showing the variation of charge on electrode $A$ versus the magnitude of force.

In this Fig. , the vertical axis is the output charge in pC and the horizontal axis is applied load in Newtons. 
The data set was fitted into a linear 6th-degree polynomial:

$c_{A}=-0.356 f^{6}+7.252 f^{5}-56.47 f^{4}+218.67 f^{3}-$

$480.4 f^{2}+1025.3 f+101.41$

Due to the symmetry in geometry and loading, charge on element $C$ is exactly the same as that on element $A$. Hence, focus is shifted on the behavior of the other two electrodes, namely, $B$ and $D$. Again, due to the symmetry considerations, just one of them, in this case element $B$, will be studied. The curves shown in Fig. 6 are associated with the data collected from studying the behavior of electrode $B$.

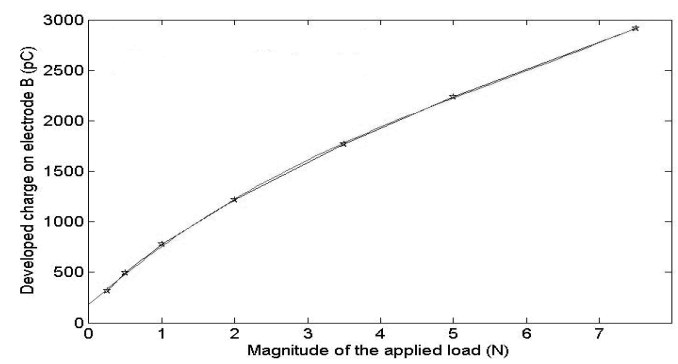

Fig. 6: Curve showing the variation of charge on electrode $B$ versus the magnitude of force.

The result of curve-fitting is:

$c_{B}=-0.339 f^{6}+6.910 f^{5}-53.752 f^{4}+207.82 f^{3}-$

$455.61 f^{2}+971.06 f+98.484$

Now back to Equation 9 and Table 4 and based on what were discussed above, Equation 9 can be cast in the following form:

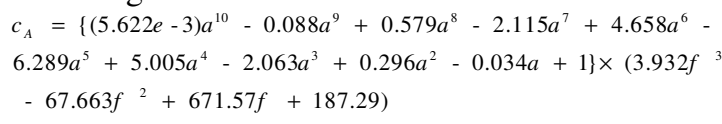

From a mathematical point of view, the above equation is a bi-variate equation which incorporates both the angle of rotation and the magnitude of the applied force. Therefore, instead of using the lookup Table 4, this equation can be used. Fig. 7 is a 3D illustration of how the charge on electrode $A$ varies with respect to the angle of rotation of rectangular probe and the force applied on it.

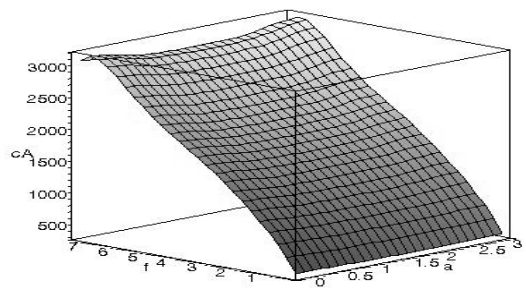

Fig. 7: Surface showing variation of charge on electrode $A$ versus rotation and force.
Following a similar discussion, the equation below and 3D surface (Fig. 8), are derived for electrode $B$ :

$c_{B}=\left\{-(5.566 e-3) a^{10}+0.088 a^{9}-0.589 a^{8}+2.129 a^{7}\right.$

$\left.-4.516 a^{6}+5.722 a^{5}-4.260 a^{4}+1.799 a^{3}-0.382 a^{2}+0.035 a+1\right\} \times$

$\left(3.744 f^{3}-64.245 f^{2}+636.42 f+179.6\right)$

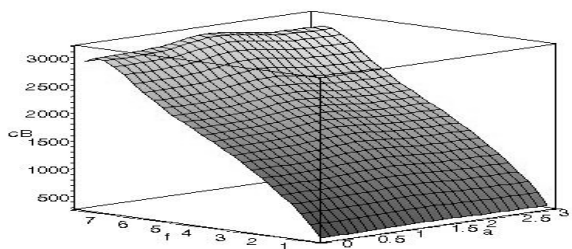

Fig. 8: Surface showing variation of charge on electrode $B$ versus rotation and force

In order to investigate the accuracy of the preceding two surfaces, as an example, each of them is intersected with the plane $f=1 \mathrm{~N}$. The resulting curves should be identical to those shown in Fig. 3. The results are shown in the following figures, i.e., Figs. s 9 and 10 .

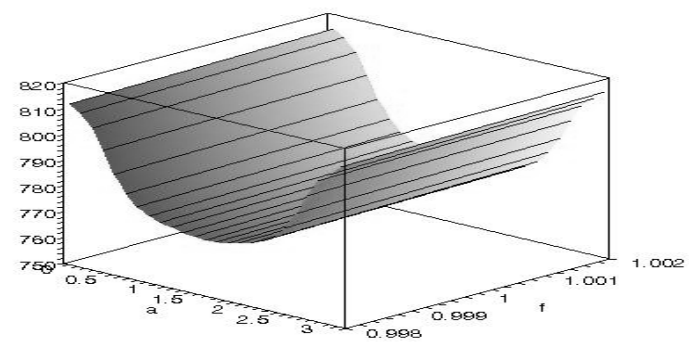

Fig. 9: Cross-section showing the charge versus angle of rotation for electrode $A$ when $f=1 \mathrm{~N}$.

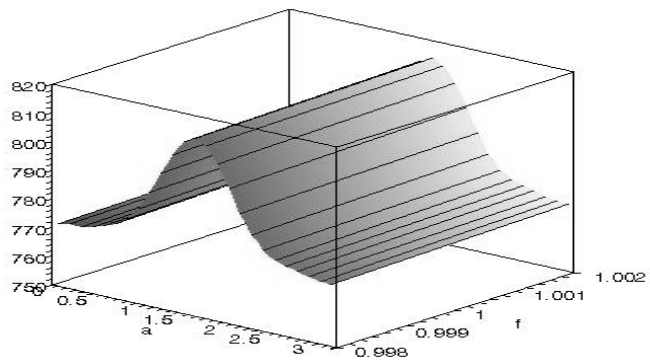

Fig. 10: Cross-section showing the charge versus angle of rotation for electrode $B$ when $f=1 \mathrm{~N}$.

Both of the cross-sections are in excellent agreement with those in Figs. 3. So, it can be concluded that curve-fitting the data with polynomials can be very accurate.

Dependency on magnitude of applied force: Another point to raise here is how the output charge on the electrodes $A$ and $B$ vary against load at different angles 
of rotation. In a set of new simulations, first the rotation angle of probe was fixed at a certain amount, and then the magnitude of applied force was varied. The probe was placed at the center of the membrane and fixed at various angles of rotation, varying in increments of $15^{\circ}$. At each of the angles, seven distinct loads were exerted, and the curves shown in Figs 11 and 12 were consecutively developed.

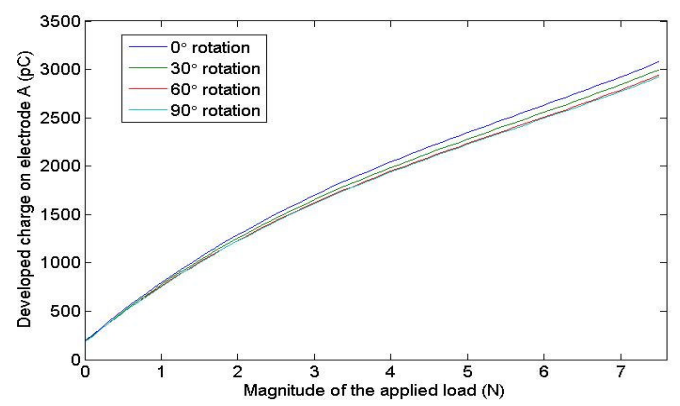

Fig. 11: Curves depicting variation of output charge on electrode $A$ versus the force at different angles.

As noticed in Fig. 11, the curves follow similar patterns. The upper-most curve corresponds to the $0^{\circ}$ angle, and the lower-most one matches the $90^{\circ}$. The other two fill in between. For electrode $B$ (Fig. 12), there is not a big difference between the curves. The only difference this time is that the uppermost and lowermost curves correspond to, respectively, $90^{\circ}$ and $0^{\circ}$ angles.

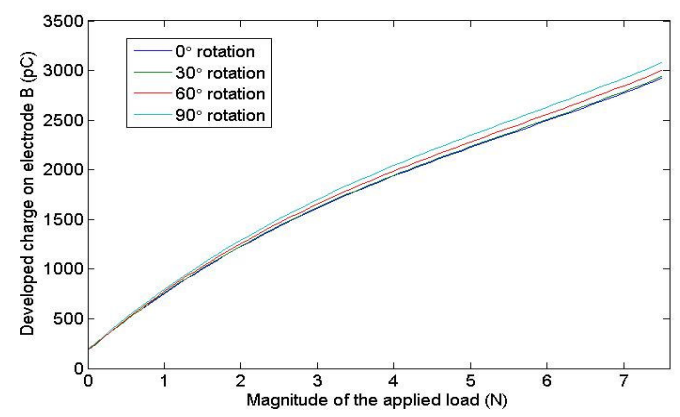

Fig. 12: Curves depicting variation of output charge on electrode $B$ versus the force at different angles.

\section{CONCLUSION}

It is concluded that by using the proposed approach the sensitivity of the sensor to the orientation of the touching object can be investigated with reasonable accuracy. Computer simulations were done by using a rectangular probe of $14 \mathrm{~mm}$ by $7 \mathrm{~mm}$ size and applying three magnitudes of forces, namely $0.5 \mathrm{~N}, 1 \mathrm{~N}$ and $2 \mathrm{~N}$. In each simulation, the magnitude of the applied force was fixed and the probe was rotated between $0^{\circ}$ to $180^{\circ}$, in increments of $10^{\circ}$, with respect to the drawn or $x$ direction of the PVDF film. The obtained charges at different angles were fitted into curves, 10th-degree polynomials. The accuracy of the obtained polynomials was verified by setting them to values in between those of the discrete data. It was noticed that for each magnitude of force, the range of variation of output electrical charge was different. However, all the curves were similar to each other in shape. Therefore, an attempt was made to find a general equation, showing the variation of output charge versus magnitude of the applied force. This was done by normalization of the polynomials. Later, the normalized equation was multiplied by another polynomial, which gave the amount of output charge versus the magnitude of the applied force. In conclusion, a bi-variate function was obtained, showing the amount of output charge against both the angle of orientation and the magnitude of the applied force. For both electrodes $A$ and $B$, surfaces were plotted based on their related bi-variate functions. Finally, a different approach was taken to investigate the dependency of the output charges on electrodes on the angles of rotation and magnitudes of the applied force. This time, first, the angle of rotation was set at a constant value, and then the magnitude of the force was changed. The results were presented in terms curves.

Work is currently underway in our lab to detect the complete shape of the touched object using the proposed method.

\section{ACKNOWLEDGEMENTS}

The authors thank the Institute for Robotics and Intelligent Systems (IRIS), and the Natural Sciences and Engineering Research Council (NSERC) of Canada for providing partial financial support. We also would like to express our gratitude to the Center of Excellence of Biomedical Engineering of Iran based in Amirkabir University of technology, Faculty of Biomedical Engineering for its contribution. We also appreciate the support provided via the grant awarded by the Ministry of Health of Iran through Razi International Festival.

\section{REFERENCES}

1. Dargahi, J., S. Najarian, 2004. Analysis of a Membrane Type Polymeric-Based Tactile Sensor for Biomedical and Medical Robotic Applications. Sensors and Materials, 16(1): 25-41.

2. Dargahi, J., S. Najarian, 2004. An Integrated Force-Position Tactile Sensor for Improving Diagnostic and Therapeutic Endoscopic Surgery. Bio-Medical Materials and Engineering, 14(2): 151-166. 
3. Najarian, S., J. Dargahi, and M. A. Changizi, 2006. A Tactile Probe with Applications in Biomedical Robotics. Proceedings of the IEEE ISIE06 Automated Conference Submission System, Montreal, Canada.

4. Dargahi, J., S. Najarian, V. Mirjalili, and B. Liu, 2007. Modeling and Testing of a Sensor Capable of Determining the Stiffness of Biological Tissues. Canadian Journal of Electrical and Computer Engineering, 32(2): 45-51.

5. Hosseini, M., S. Najarian, S. Motaghinasab, and J. Dargahi, 2006. Detection of Tumors using Computational Tactile Sensing Approach. International Journal of Medical Robotics and Computer Assisted Surgery, 2(4): 333-340.

6. Dargahi, J., S. Najarian, and B. Liu, 2007. Sensitivity Analysis of a Novel Tactile Probe for Measurement of Tissue Softness with Applications in Biomedical Robotics. Journal of Materials Processing Technology, 183: 176-182.

7. Mirbagheri, A., J. Dargahi, S. Najarian, and F. Tabatabai Ghomshe, 2007. Design, Fabrication, and Testing of a Membrane Piezoelectric Tactile Sensor with Four Sensing Elements. American Journal of Applied Sciences, 4(9): 645-652.

8. Ramezanifard, R., J. Dargahi, S. Najarian, and F. Tabatabai Ghomshe, 2007. A Novel Modeling Approach for Collision Avoidance in Robotic Surgery. American Journal of Applied Sciences, 4(9): 693-699.

9. Amiri, M., S. M. Hosseini, and S. Najarian, 2007. Application of Artificial Neural Networks for Estimation of Tumor Parameters in Biological Tissues. Proceedings of the 3rd International Conference on Natural Computation (ICNC'07), Haikou, China.

10. Dargahi, J., S. Najarian, 2004. Theoretical and Experimental Analysis of a Piezoelectric Tactile Sensor for Use in Endoscopic Surgery. Sensor Review, 24(1): 74-83.

11. Bicchi, A., G. Canepa, D.De. Rossi, P. Iacconi, E.P. Scilingo, 1996. A Sensorized Minimally Invasive Surgery Tool for Detecting Tissue Elastic Properties. Proceedings of IEEE International Conference Robotics and Automation, Minneapolis, USA, 884-888.

12. Brouwer, I., J. Ustin, L. Bentley, A. Sherman, N. Dhruv, F. Tendick, 2001. Measuring in vivo Animal Soft Tissue Properties for Haptic Modeling in Surgical Simulation. In Studies in Health Technology Informatics - Medicine Meets Virtual Reality, Amsterdam: ISO Press, 69-74.
13. Dargahi, J., S. Najarian, 2004. A Supported Membrane Type Sensor for Medical Tactile Mapping. Sensor Review, 24(3): 284-297.

14. Hannaford, B., J. Trujillo, M. Sinanan, M. Moreyra, J. Rosen, J. Brown, R. Leuschke, M. MacFarlane, 1998. Computerized Endoscopic Surgical Grasper. In Studies in Health Technology Informatics-Medicine Meets Virtual Reality, Amsterdam, ISO Press, 265-271.

15. Dargahi, J., S. Najarian, 2004. Human Tactile Perception as a Standard for Artificial Tactile Sensing-a review. International Journal of Medical Robotics and Computer Assisted Surgery, 1(13): 23-35.

16. Dargahi, J., S. Najarian, 2005. Advances in Tactile Sensors Design/Manufacturing and Its Impact on Robotics Applications- a review. Industrial Robot, 32(3): 268-281.

17. Dargahi, J., S. Najarian, 2003. An Endoscopic Force Position Grasper with Minimum Sensors. Canadian Journal of Electrical and Computer Engineering, 28(3/4): 155-161.

18. Dargahi, J., S. Najarian, X.Z. Zheng, 2005. Measurements and Modeling of Compliance Using a Novel Multi-Sensor Endoscopic Grasper Device. Sensors and Materials, 17(1): 7-20.

19. Bar-Cohen, Y., C. Mavroidis, M. Bouzit, B. Dolgin, D. Harm, G. Kopchok, R. White, 2000. Virtual Reality Robotic Operation Simulations Using MEMICA Haptic System. Proc. Int. Conf. for Smart Systems and Robotics for Medicine and Space Applications, Houston, USA.

20. Dargahi, J., M. Parameswaran, S. Payandeh, 2000. A Micromachined Piezoelectric Tactile Sensor for Endoscopic Grasper-Theory, Fabrication, and Experiments. Journal of Microelectromechanical Systems, 9(3): 329-335.

21. Odon, A., 2003. Probe with PVDF Sensor for Energy Measurements of Optical Radiation. Measurement Science Review, 3: 111-114.

22. Murayamaa, Y., C. E. Constantinoub, S. Omataa, 2004. Micro-mechanical Sensing Platform for the Characterization of the Elastic Properties of the Ovum via Uniaxial Measurement. Journal of Biomechanics, 37: 67-72.

23. Brady, S., K.T. Lau, W. Megill, G.G. Wallace, D. Diamond, 2005. Development and Characterization of Conducting Polymeric-based Sensing Devices. Synthetic Metals, 154: 25-28. 